\title{
Persistence and non-persistence of a stochastic food chain model with finite delay
}

\author{
Haihong $\mathrm{Li}^{\mathrm{a}, *}$, Haixia $\mathrm{Li}^{\mathrm{b}}$, Fuzhong Cong ${ }^{\mathrm{c}}$ \\ ${ }^{a}$ Department of Basic Science, Jilin Construction University, Changchun 130024, China. \\ ${ }^{b}$ School of Business, Changchun Guanghua University, Changchun 130024, China. \\ ${ }^{c}$ Department of Basic Courses, Air Force Aviation University, Changchun 130022, Jilin, China.
}

Communicated by A. Atangana

\begin{abstract}
In this paper, we study a three species predator-prey time-delay chain model with stochastic perturbation. First, we analyze that this system has a unique positive solution. Then, we deduce the conditions that the system is persistent in time average. After that, conditions for the system going to be extinction in probability are established. At last, numerical simulations are carried out to support our results. (C)2017 All rights reserved.
\end{abstract}

Keywords: Stochastic differential equation, persistent, non-persistent, extinction.

2010 MSC: 93E03.

\section{Introduction}

The most exciting modern application of mathematics is used in biology. As we know, two species systems such as predator-prey, plant-pest systems etcetera has been one of the dominant theme in the importance of ecology and mathematics because of its widespread. After that, the predator-prey chain model is the typical representative.

As we know, until the late 70s, some interest mathematics tritrophic food chain model appeared $[5,6]$. One of the most famous population dynamics model on ecological system has received a lot of attention and extensive research which named Lotka-Volterra predator-prey system, refer to [3, 10, 20]. Especially the persistence and extinction of this model is very interesting topic.

The three species predator-prey chain model is described as follows:

$$
\left\{\begin{array}{l}
\dot{x_{1}}(t)=x_{1}(t)\left(a_{1}-b_{11} x_{1}(t)-b_{12} x_{2}(t)\right), \\
\dot{x_{2}}(t)=x_{2}(t)\left(-a_{2}+b_{21} x_{1}(t)-b_{22} x_{2}(t)-b_{23} x_{3}(t)\right), \\
\dot{x_{3}}(t)=x_{3}(t)\left(-a_{3}+b_{32} x_{2}(t)-b_{33} x_{3}(t)\right),
\end{array}\right.
$$

\footnotetext{
*Corresponding author

Email address: lihaihong888999@126.com (Haihong Li)
}

doi:10.22436/jnsa.010.06.38 
where $x_{i}(t)(i,=1,2,3)$ represent the densities of prey, mid-level predator, and top predator species at time $t$, respectively. The parameters $a_{1}, a_{2}, a_{3}, b_{i i}(i,=1,2,3)$ are positive constants that stand for intrinsic growth rate, predator death rate of the second species, predator death rate of the third species, and coefficient of internal competition, respectively. $b_{21}, b_{32}$ represent saturated rate of the second and the third predator, $b_{12}, b_{23}$ represent the decrement rate of predator to prey. System (1.1) describes a three species predator-prey chain model in which the latter preys on the former. From a biological viewpoint, we not only require the positive solution of the system but also require its unexploded property in any finite time and stability.

We know that the global asymptotic stability of a positive equilibrium $x^{*}=\left(x_{1}^{*}, x_{2}^{*}, x_{3}^{*}\right)$ holds and is global stable if the following condition holds:

$$
a_{1}-\frac{b_{11}}{b_{21}} a_{2}-\frac{b_{11} b_{22}+b_{12} b_{21}}{b_{21} b_{32}} a_{3}>0
$$

which could refer to [9].

In a recent period of time, it is easy to understand in the process of both natural and man-made involve the time delay, such as biology, medicine and so on. Kuang [19] mentioned that animals must take time to digest their food before further activities and responses take place. So, the dynamic model of any species lack of delay is an approximate model. Standard for three classes dynamic model of a single species with independent discrete delay to have global asymptotic stability equilibrium point was set up by friedman and Gopalsamy [4]. By constructing proper lyapunov functionals, the global stability of the time-delay systems is studied by Xiaoqing et al. [28]. Hence, we introduce time-delays in system (1.1) and suppose that the middle carnivore specie needs time $\tau$ to have the ability to hunt after birth and it just captures prey and $\tau$ mature adult. Similarly, suppose that the top carnivore specie needs time $\tau$ to have the ability to hunt after birth and it just captures mid-level and $\tau([8,14,22])$ mature adult predator. Then we get

$$
\left\{\begin{array}{l}
\dot{x_{1}}(t)=x_{1}(t)\left(a_{1}-b_{11} x_{1}(t)-b_{12} x_{2}(t-\tau)\right) \\
\dot{x_{2}}(t)=x_{2}(t)\left(-a_{2}+b_{21} x_{1}(t-\tau)-b_{22} x_{2}(t)-b_{23} x_{3}(t-\tau)\right) \\
\dot{x_{3}}(t)=x_{3}(t)\left(-a_{3}+b_{32} x_{2}(t-\tau)-b_{33} x_{3}(t)\right)
\end{array}\right.
$$

However, population dynamic system in the real environment is unavoidable influenced by environmental noise (see, e.g., $[7,8])$. The parameters in the system are not absolute constants, they are always in some near average. So we can not ignore the influence of noise on the system. Recently many authors have discussed population systems subject to white noise (see, e.g., [12, 13, 15-17, 23, 29]). May (see, e.g., [25]) pointed out that due to continuous fluctuation in the environment, the equilibrium distribution does not reach a stable value, but fluctuates randomly near the average value.

Therefore, Lotka-Volterra predator-prey chain models in random environments are becoming popular. Ji et al. $[15,16]$ investigated the asymptotic behavior of the stochastic predator-prey system with perturbation. Polansky [26] and Barra et al. [1] have given some special systems of their invariant distribution. After that, Gard [9] analysed that under some conditions the stochastic food chain model exists an invariant distribution. Mao et al. [24] have discussed nonexplosion, persistence, and asymptotic stability of the stochastic delay equations, they pointed out that the noise will not only suppress a potential population explosion in the delay model but will also make the population to be stochastically ultimately bounded. However, seldom people investigate the persistent and non-persistent of the food chain time-delay model with stochastic perturbation. Li et al. have studied the food chain model with stochastic perturbation in [21], and this paper is a continuation of the previous article.

In this paper, we introduce the white noise into the intrinsic growth rate of system (1.2), and suppose $a_{i} \rightarrow a_{i}+\sigma_{i} \dot{B}_{i}(t)(i=1,2,3)$, then we obtain the following stochastic system

$$
\left\{\begin{array}{l}
d x_{1}(t)=x_{1}(t)\left(a_{1}-b_{11} x_{1}(t)-b_{12} x_{2}(t-\tau)\right) d t+\sigma_{1} x_{1}(t) d B_{1}(t), \\
d x_{2}(t)=x_{2}(t)\left(-a_{2}+b_{21} x_{1}(t-\tau)-b_{22} x_{2}(t)-b_{23} x_{3}(t-\tau)\right) d t-\sigma_{2} x_{2}(t) d B_{2}(t), \\
d x_{3}(t)=x_{3}(t)\left(-a_{3}+b_{32} x_{2}(t-\tau)-b_{33} x_{3}(t)\right) d t-\sigma_{3} x_{3}(t) d B_{3}(t),
\end{array}\right.
$$


where $B_{i}(t)(i=1,2,3)$ are independent white noises with $B_{i}(0)=0, \sigma_{i}^{2}>0(i=1,2,3)$ representing the intensities of the noise.

The aim of this paper is to discuss the long time behavior of system (1.3) by stochastic comparison theorem which is different from Mao et al. [24]. We have mentioned that $x^{*}=\left(x_{1}^{*}, x_{2}^{*}, x_{3}^{*}\right)$ is also the positive equilibrium of system (1.2). But, when it is suffered stochastic perturbations, there is no positive equilibrium. Hence, it is impossible that the solution of system (1.3) will tend to a fixed point. In this paper, we show that system (1.3) is persistent in time average. Furthermore, under certain conditions, we prove the population of system (1.3) will die out in probability which will not happen in deterministic system and could reveal that large white noise may lead to extinction.

The rest of this paper is organized as follows. In Section 2, we show that there is a unique non-negative solution of system (1.3). In Section 3, we show that system (1.3) is persistent in time average. While in Section 4, we consider three situations when the population of the system will be extinction. In Section 5, numerical simulations are carried out to support our results.

Throughout this paper, unless otherwise specified, let $\left(\Omega,\left\{\mathcal{F}_{t}\right\}_{t} \geqslant 0, P\right)$ be a complete probability space with a filtration $\left\{\mathcal{F}_{t}\right\}_{t \geqslant 0}$ satisfying the usual conditions (i.e., it is right continuous and $\mathcal{F}_{0}$ contains all P-null sets). Let $R_{+}^{3}$ denote the positive cone of $R^{3}$, namely $R_{+}^{3}=\left\{x \in R^{3}: x_{i}>0,1 \leqslant i \leqslant 3\right\}, \bar{R}_{+}^{3}=\{x \in$ $\left.R^{3}: x_{i} \geqslant 0,1 \leqslant i \leqslant 3\right\}$.

\section{Existence and uniqueness of the nonnegative solution}

To investigate the dynamical behavior, the first concern thing is whether the solution is global existence. Moreover, for a population model, whether the solution is nonnegative is also considered. Hence, in this section we show that the solution of system (1.3) is global and nonnegative. As we have known, in order for a stochastic differential equation to have a unique global (i.e., no explosion at a finite time) solution with any given initial value, the coefficients of the equation are generally required to satisfy the linear growth condition and local Lipschitz condition (see, e.g., [22]). It is easy to see that the coefficients of system (1.3) are locally Lipschitz continuous, so system (1.3) has a local solution. By Theorem 2.2, we show the global existence of this solution.

Let $N(t)$ be the solution of the non-autonomous logistic equation with random perturbation

$$
d N(t)=N(t)[(a(t)-b(t) N(t)) d t+\alpha(t) d B(t)],
$$

where $B(t)$ is one-dimensional standard Brownian motion, $N(0)=N_{0}>0$ and $N_{0}$ is independent of $B(t)$.

Lemma 2.1 ([18]). There exists a unique continuous solution $\mathrm{N}(\mathrm{t})$ of $(2.1)$ for any initial value $\mathrm{N}(0)=\mathrm{N}_{0}>0$, which is global and represented by

$$
\mathrm{N}(\mathrm{t})=\frac{\exp \left\{\int_{0}^{\mathrm{t}}\left[\mathrm{a}(\mathrm{s})-\frac{\sigma^{2}(\mathrm{~s})}{2}\right] \mathrm{ds}+\sigma(\mathrm{s}) \mathrm{dB}(\mathrm{s})\right\}}{1 / \mathrm{N}_{0}+\int_{0}^{\mathrm{t}} \mathrm{b}(\mathrm{s}) \exp \left\{\int_{0}^{s}\left[\mathrm{a}(\tau)-\frac{\sigma^{2}(\tau)}{2}\right] \mathrm{d} \tau+\sigma(\tau) \mathrm{dB}(\tau)\right\} \mathrm{ds}}, \mathrm{t} \geqslant 0 .
$$

In order to get the conclusion, we should introduce two systems first,

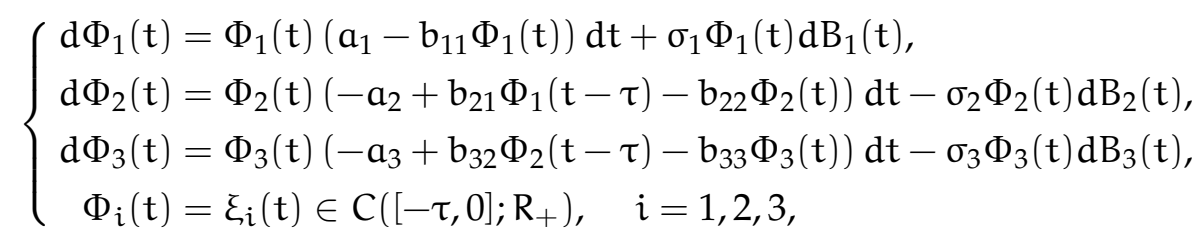

and

$$
\left\{\begin{aligned}
d I_{1}(t) & =I_{1}(t)\left(a_{1}-b_{11} I_{1}(t)-b_{12} \Phi_{2}(t-\tau)\right) d t+\sigma_{1} I_{1}(t) d B_{1}(t), \\
d I_{2}(t) & =I_{2}(t)\left(-a_{2}+b_{21} I_{1}(t-\tau)-b_{22} I_{2}(t)-b_{23} \Phi_{3}(t-\tau)\right) d t-\sigma_{2} I_{2}(t) d B_{2}(t), \\
d I_{3}(t) & =I_{3}(t)\left(-a_{3}+b_{32} I_{2}(t-\tau)-b_{33} I_{3}(t)\right) d t-\sigma_{3} I_{3}(t) d B_{3}(t), \\
I_{i}(t) & =\xi_{i}(t) \in C\left([-\tau, 0] ; R_{+}\right) \quad i=1,2,3,
\end{aligned}\right.
$$


where

$$
\Phi(\mathrm{t})=\left(\Phi_{1}(\mathrm{t}), \Phi_{2}(\mathrm{t}), \Phi_{3}(\mathrm{t})\right)^{\top}, \quad \mathrm{I}(\mathrm{t})=\left(\mathrm{I}_{1}(\mathrm{t}), \mathrm{I}_{2}(\mathrm{t}), \mathrm{I}_{3}(\mathrm{t})\right)^{\top},
$$

are the solutions of the above stochastic differential equations with time delay.

Theorem 2.2. For any initial data $x(t)=\left\{\left(\xi_{1}(t), \xi_{2}(t), \xi_{3}(t)\right):-\tau \leqslant t \leqslant 0\right\} \in C\left([-\tau, 0] ; R_{+}^{3}\right)$, the positive solution of system (1.3) has the property that

$$
I(t) \leqslant x(t) \leqslant \Phi(t),
$$

i.e.,

$$
\mathrm{I}_{\mathfrak{i}}(\mathrm{t}) \leqslant \mathrm{x}_{\mathfrak{i}}(\mathrm{t}) \leqslant \Phi_{\mathfrak{i}}(\mathrm{t}), \quad \mathfrak{i}=1,2,3,
$$

where

$$
\Phi(t)=\left(\Phi_{1}(t), \Phi_{2}(t), \Phi_{3}(t)\right)^{\top}, \quad I(t)=\left(I_{1}(t), I_{2}(t), I_{3}(t)\right)^{\top},
$$

are solutions of systems (2.3) and (2.4) stochastic differential equations with time delay.

Proof. Let $z_{1}(t)=\frac{1}{x_{1}(t)}$. Then, by Itô's formula, we have

$$
\begin{aligned}
d z_{1}(t)=d\left(\frac{1}{x_{1}(t)}\right) & =-\left[\left(\frac{a_{1}}{x_{1}(t)}-\frac{b_{12} x_{2}(t-\tau)}{x_{1}(t)}-b_{11}\right) d t+\frac{\sigma_{1}}{x_{1}(t)} d B_{1}(t)\right]+\frac{\sigma_{1}^{2}}{x_{1}(t)} d t \\
& =\left[\left(\sigma_{1}^{2}-a_{1}\right) z_{1}(t)+b_{11}+\frac{b_{12} x_{2}(t-\tau)}{x_{1}(t)}\right] d t-\sigma_{1} z_{1}(t) d B_{1}(t) \\
& =\left[\left(\sigma_{1}^{2}-a_{1}\right) d t-\sigma_{1} d B_{1}(t)\right] z_{1}(t)+\left(b_{11}+\frac{b_{12} x_{2}(t-\tau)}{x_{1}(t)}\right) d t .
\end{aligned}
$$

That is,

$$
d z_{1}(t)=\left[\left(\sigma_{1}^{2}-a_{1}\right) d t-\sigma_{1} d B_{1}(t)\right] z_{1}(t)+\left(b_{11}+\frac{b_{12} x_{2}(t-\tau)}{x_{1}(t)}\right) d t
$$

Then

$$
\begin{aligned}
z_{1}(t) & =e^{\int_{0}^{t}\left(\frac{\sigma_{1}^{2}}{2}-a_{1}\right) d s-\sigma_{1} d B_{1}(s)}\left[\frac{1}{x_{1}(0)}+\int_{0}^{t}\left(b_{11}+\frac{b_{12} x_{2}(t-\tau)}{x_{1}(t)}\right) e^{\int_{0}^{s}\left(a_{1}-\frac{\sigma_{1}^{2}}{2}\right) d \tau+\sigma_{1} d B_{1}(\tau)} d s\right] \\
& =e^{\left(\frac{\sigma_{1}^{2}}{2}-a_{1}\right) t-\sigma_{1} B_{1}(t)}\left[\frac{1}{x_{1}(0)}+\int_{0}^{t}\left(b_{11}+\frac{b_{12} x_{2}(t-\tau)}{x_{1}(t)}\right) e^{\left(a_{1}-\frac{\sigma_{1}^{2}}{2}\right) s+\sigma_{1} B_{1}(s)} d s\right] \\
& \geqslant e^{\left(\frac{\sigma_{1}^{2}}{2}-a_{1}\right) t-\sigma_{1} B_{1}(t)}\left[\frac{1}{x_{1}(0)}+\int_{0}^{t} b_{11} e^{\left(a_{1}-\frac{\sigma_{1}^{2}}{2}\right) s+\sigma_{1} B_{1}(s)} d s\right] \\
& =\Phi_{1}^{-1}(t) .
\end{aligned}
$$

By Lemma 2.1, we obtain that $\Phi_{1}(t)$ is the solution of the following equation

$$
d \Phi_{1}(t)=\Phi_{1}(t)\left(a_{1}-b_{11} \Phi_{1}(t)\right) d t+\sigma_{1} \Phi_{1}(t) d B_{1}(t) .
$$

Hence, we have

$$
x_{1}(t) \leqslant \Phi_{1}(t), \text { a.s.. }
$$


On the other hand, let $z_{2}(t)=\frac{1}{x_{2}(t)}$. Then, by Itô's formula, we could derive that

$$
\begin{aligned}
d z_{2}(t)=d\left(\frac{1}{x_{2}(t)}\right) & =-\left[\left(-\frac{a_{2}}{x_{2}(t)}+\frac{b_{21} x_{1}(t-\tau)}{x_{2}(t)}-b_{22}-\frac{b_{23} x_{3}(t-\tau)}{x_{2}(t)}\right) d t-\frac{\sigma_{2}}{x_{2}(t)} d B_{2}(t)\right]+\frac{\sigma_{2}^{2}}{x_{2}(t)} d t \\
& =\left[\left(\sigma_{2}^{2}+a_{2}\right) z_{2}(t)+b_{22}-b_{21} x_{1}(t-\tau) z_{2}(t)-b_{23} x_{3}(t-\tau) z_{2}(t)\right] d t+\sigma_{2} z_{2}(t) d B_{2}(t) \\
& =\left[\left(\sigma_{2}^{2}+a_{2}-b_{21} x_{1}(t-\tau)-b_{23} x_{3}(t-\tau)\right) d t+\sigma_{2} d B_{2}(t)\right] z_{2}(t)+b_{22} d t
\end{aligned}
$$

then

$$
\begin{aligned}
z_{2}(t)= & \frac{1}{x_{2}(0)} e^{\left(\frac{\sigma_{2}^{2}}{2}+a_{2}\right) t+\sigma_{2} B_{2}(s)-b_{21} \int_{0}^{t} x_{1}(s-\tau) d s+b_{23} \int_{0}^{t} x_{3}(s-\tau) d s} \\
& +b_{22} \int_{0}^{t} e^{\left(a_{2}+\frac{\sigma_{2}^{2}}{2}\right)(t-s)+\sigma_{2}\left(B_{2}(t)-B_{2}(s)\right)-b_{21} \int_{s}^{t} x_{1}(\mu-\tau) d \mu+b_{23} \int_{s}^{t} x_{3}(\mu-\tau) d \mu} d s \\
\geqslant & \frac{1}{x_{2}(0)} e^{\left(\frac{\sigma_{2}^{2}}{2}+a_{2}\right) t+\sigma_{2} B_{2}(s)-b_{21} \int_{0}^{t} x_{1}(s-\tau) d s} \\
& +b_{22} \int_{0}^{t} e^{\left(a_{2}+\frac{\sigma_{2}^{2}}{2}\right)(t-s)+\sigma_{2}\left(B_{2}(t)-B_{2}(s)\right)-b_{21} \int_{s}^{t} \Phi_{1}(\mu-\tau) d \mu} d s \\
= & \Phi_{2}^{-1}(t) .
\end{aligned}
$$

Therefore

$$
x_{2}(t) \leqslant \Phi_{2}(t), \text { a.s.. }
$$

By Lemma 2.1, we obtain that $\Phi_{2}(t)$ is the solution of the following equation

$$
d \Phi_{2}(t)=\Phi_{2}(t)\left(-a_{2}+b_{21} \Phi_{1}(t-\tau)-b_{22} \Phi_{2}(t)\right) d t-\sigma_{2} \Phi_{2}(t) d B_{2}(t) .
$$

At last, let $z_{3}(t)=\frac{1}{x_{3}(t)}$. Then, by Itô's formula, we could derive that

$$
\begin{aligned}
d z_{3}(t)=d\left(\frac{1}{x_{3}(t)}\right) & =-\left[\left(-\frac{a_{3}}{x_{3}(t)}+\frac{b_{32} x_{2}(t-\tau)}{x_{3}(t)}-b_{33}\right) d t-\frac{\sigma_{3}}{x_{3}(t)} d B_{3}(t)\right]+\frac{\sigma_{3}^{2}}{x_{3}(t)} d t \\
& =\left[\left(\sigma_{3}^{2}+a_{3}\right) z_{2}(t)+b_{33}-b_{32} x_{2}(t-\tau) z_{3}(t)\right] d t+\sigma_{3} z_{3}(t) d B_{3}(t) \\
& =\left[\left(\sigma_{3}^{2}+a_{3}-b_{32} x_{2}(t-\tau)\right) d t+\sigma_{2} d B_{3}(t)\right] z_{2}(t)+b_{33} d t
\end{aligned}
$$

then

$$
\begin{aligned}
z_{3}(t)= & \frac{1}{x_{3}(0)} e^{\left(\frac{\sigma_{3}^{2}}{2}+a_{3}\right) t+\sigma_{3} B_{3}(s)-b_{32} \int_{0}^{t} x_{2}(s-\tau) d s} \\
& +b_{33} \int_{0}^{t} e^{\left(a_{3}+\frac{\sigma_{3}^{2}}{2}\right)(t-s)+\sigma_{3}\left(B_{3}(t)-B_{3}(s)\right)-b_{32} \int_{s}^{t} x_{2}(\mu-\tau) d \mu} d s \\
\geqslant & \frac{1}{x_{3}(0)} e^{\left(\frac{\sigma_{3}^{2}}{2}+a_{3}\right) t+\sigma_{3} B_{3}(s)-b_{32} \int_{0}^{t} \Phi_{2}(s-\tau) d s} \\
& +b_{33} \int_{0}^{t} e^{\left(a_{3}+\frac{\sigma_{3}^{2}}{2}\right)(t-s)+\sigma_{3}\left(B_{3}(t)-B_{3}(s)\right)-b_{32} \int_{s}^{t} \Phi_{2}(\mu-\tau) d \mu} d s \\
= & \Phi_{3}^{-1}(t),
\end{aligned}
$$

then, it is easy to see that $\Phi_{3}(t)$ is the solution of the following equation

$$
d \Phi_{3}(t)=\Phi_{3}(t)\left(-a_{3}+b_{32} \Phi_{2}(t-\tau)-b_{33} \Phi_{3}(t)\right) d t-\sigma_{3} \Phi_{3}(t) d B_{3}(t),
$$


and

$$
x_{3}(t) \leqslant \Phi_{3}(t) \text {, a.s.. }
$$

In the same method, we could derive that

$$
x_{\mathfrak{i}}(t) \geqslant I_{i}(t) \quad \text { a.s., } i=1,2,3,
$$

where $I(t)=\left(I_{1}(t), I_{2}(t), I_{3}(t)\right){ }^{\top}$ is the solution of system (2.4).

Remark 2.3. From Lemma 2.1, we know

$$
\begin{aligned}
& \frac{1}{\Phi_{1}(t)}=\frac{1}{x_{1}(0)} e^{\left(\frac{\sigma_{1}^{2}}{2}-a_{1}\right) t-\sigma_{1} B_{1}(t)}+b_{11} \int_{0}^{t} e^{\left(\frac{\sigma_{1}^{2}}{2}-a_{1}\right)(t-s)-\sigma_{1}\left(B_{1}(t)-B_{1}(s)\right)} d s, \\
& \frac{1}{\Phi_{2}(t)}=\frac{1}{x_{2}(0)} e^{\left(\frac{\sigma_{2}^{2}}{2}+a_{2}\right) t+\sigma_{2} B_{2}(t)-b_{21} \int_{0}^{t} \Phi_{1}(s-\tau) d s}+b_{22} \int_{0}^{t} e^{\left(\frac{\sigma_{2}^{2}}{2}+a_{2}\right)(t-s)+\sigma_{2}\left(B_{2}(t)-B_{2}(s)\right)-b_{21} \int_{s}^{t} \Phi_{1}(\mu-\tau) d \mu} d s, \\
& \frac{1}{\Phi_{3}(t)}=\frac{1}{x_{3}(0)} e^{\left(\frac{\sigma_{3}^{2}}{2}+a_{3}\right) t+\sigma_{3} B_{3}(t)-b_{32} \int_{0}^{t} \Phi_{2}(s-\tau) d s}+b_{33} \int_{0}^{t} e^{\left(\frac{\sigma_{3}^{2}}{2}+a_{3}\right)(t-s)+\sigma_{3}\left(B_{3}(t)-B_{3}(s)\right)-b_{32} \int_{s}^{t} \Phi_{2}(\mu-\tau) d \mu} d s ;
\end{aligned}
$$

and

$$
\begin{aligned}
\frac{1}{\mathrm{I}_{1}(\mathrm{t})}= & \frac{1}{\chi_{1}(0)} e^{\left(\frac{\sigma_{1}^{2}}{2}-a_{1}\right) t-\sigma_{1} B_{1}(t)+b_{12} \int_{0}^{t} \Phi_{2}(s-\tau) d s}+b_{11} \int_{0}^{t} e^{\left(\frac{\sigma_{1}^{2}}{2}-a_{1}\right)(t-s)-\sigma_{1}\left(B_{1}(t)-B_{1}(s)\right)+b_{12} \int_{0}^{t} \Phi_{2}(\mu-\tau) d \mu} d s, \\
\frac{1}{I_{2}(t)}= & \frac{1}{x_{2}(0)} e^{\left(\frac{\sigma_{2}^{2}}{2}+a_{2}\right) t+\sigma_{2} B_{2}(t)-b_{21} \int_{0}^{t} I_{1}(s-\tau) d s+b_{23} \int_{0}^{t} \Phi_{3}(s-\tau) d s} \\
& +b_{22} \int_{0}^{t} e^{\left(\frac{\sigma_{2}^{2}}{2}+a_{2}\right)(t-s)+\sigma_{2}\left(B_{2}(t)-B_{2}(s)\right)-b_{21} \int_{s}^{t} I_{1}(\mu-\tau) d \mu+b_{23} \int_{0}^{t} \Phi_{3}(\mu-\tau) d \mu} d s, \\
\frac{1}{I_{3}(t)}= & \frac{1}{x_{3}(0)} e^{\left(\frac{\sigma_{3}^{2}}{2}+a_{3}\right) t+\sigma_{3} B_{3}(t)-b_{32} \int_{0}^{t} I_{2}(s-\tau) d s}+b_{33} \int_{0}^{t} e^{\left(\frac{\sigma_{3}^{2}}{2}+a_{3}\right)(t-s)+\sigma_{3}\left(B_{3}(t)-B_{3}(s)\right)-b_{32} \int_{s}^{t} I_{2}(\mu-\tau) d \mu} d s .
\end{aligned}
$$

From the representations of $\Phi_{i}(t)$ and $I_{i}(t),(i=1,2,3)$, Theorem 2.2 tells us the species will not reach zero in finite time.

From now on, we denote the unique global positive solution of system (1.3) with the given initial data $\xi=\left\{\xi(t)=\left(\xi_{1}(t), \xi_{2}(t), \xi_{3}(t)\right):-\tau \leqslant t \leqslant 0\right\} \in C\left([-\tau, 0] ; R_{3}^{+}\right)$by $x(t, \xi)$. In the same way, we define the solutions of systems $(2.3)$ and $(2.4)$ by $\Phi(t, \xi), I(t, \xi)$.

\section{Persistent in time average}

There is no equilibrium of system (1.3). Hence we can not show the permanence of the system by proving the stability of the positive equilibrium as the deterministic system. In this section we first show that this system is persistent in mean. Before we give the result, we should do some preparation work.

Chen et al. in [2] proposed the definition of persistence in mean for the deterministic system. Here, we also use this definition for the stochastic system.

Definition 3.1. System (1.3) is said to be persistent in mean, if

$$
\liminf _{t \rightarrow \infty} \frac{1}{t} \int_{0}^{t} x_{3}(s) d s>0, \text { a.s.. }
$$

Before giving the result, we do some preparation work. 
Lemma 3.2 ([27]). Let $\mathrm{f} \in \mathrm{C}([0,+\infty) \times \Omega,(0,+\infty)), \mathrm{F} \in \mathrm{C}([0,+\infty) \times \Omega, \mathrm{R})$. If there exist positive constants $\lambda_{0}, \lambda$, such that

$$
\log f(t) \geqslant \lambda t-\lambda_{0} \int_{0}^{t} f(s) d s+F(t), \quad t \geqslant 0 \quad \text { a.s. }
$$

and $\lim _{t \rightarrow \infty} \frac{F(t)}{t}=0$ a.s., then

$$
\liminf _{t \rightarrow \infty} \frac{1}{t} \int_{0}^{t} f(s) d s \geqslant \frac{\lambda}{\lambda_{0}}, \text { a.s.. }
$$

From Lemma 3.2, it is easy to see that we could get Lemmas 3.3 and 3.4 with the same method.

Lemma 3.3. Let $f \in C([0,+\infty) \times \Omega,(0,+\infty)), F \in C([0,+\infty) \times \Omega, R)$. If there exist positive constants $\lambda_{0}, \lambda$, such that

$$
\log f(t) \leqslant \lambda t-\lambda_{0} \int_{0}^{t} f(s) d s+F(t), \quad t \geqslant 0, \quad \text { a.s. },
$$

and $\lim _{t \rightarrow \infty} \frac{F(t)}{t}=0$, a.s., then

$$
\limsup _{t \rightarrow \infty} \frac{1}{t} \int_{0}^{t} f(s) d s \leqslant \frac{\lambda}{\lambda_{0}}, \text { a.s.. }
$$

Lemma 3.4. Let $f \in C([0,+\infty) \times \Omega,(0,+\infty)), F \in C([0,+\infty) \times \Omega, R)$. If there exist positive constants $\lambda_{0}, \lambda$, such that

$$
\log f(t)=\lambda t-\lambda_{0} \int_{0}^{t} f(s) d s+F(t), \quad t \geqslant 0, \quad \text { a.s., }
$$

and $\lim _{t \rightarrow \infty} \frac{F(t)}{t}=0$, a.s., then

$$
\lim _{t \rightarrow \infty} \frac{1}{t} \int_{0}^{t} f(s) d s=\frac{\lambda}{\lambda_{0}}, \text { a.s.. }
$$

Assumption 3.5.

$$
r_{1}-\frac{b_{11}}{b_{21}} r_{2}-\frac{b_{11} b_{22}+b_{12} b_{21}}{b_{21} b_{32}} r_{3}>0, \quad r_{1}=a_{1}-\frac{\sigma_{1}^{2}}{2}>0, \quad r_{i}=a_{i}+\frac{\sigma_{i}^{2}}{2} i=2,3 .
$$

Lemma 3.6. If Assumption 3.5 is satisfied, then the solution $\Phi(t, \xi)$ of system (2.2) has the following property:

$$
\lim _{\mathfrak{t} \rightarrow \infty} \frac{\log \Phi_{\mathfrak{i}}(\mathrm{t})}{\mathrm{t}}=0, \quad \lim _{\mathrm{t} \rightarrow \infty} \frac{1}{\mathrm{t}} \int_{0}^{\mathrm{t}} \Phi_{\mathfrak{i}}(\mathrm{s}) \mathrm{d} s=M_{\mathrm{i}}, \quad \text { a.s., }
$$

where

$$
M_{1}=\frac{r_{1}}{b_{11}}, \quad M_{2}=\frac{r_{1} b_{21}-r_{2} b_{11}}{b_{11}}, \quad M_{3}=\frac{r_{1} b_{21} b_{32}-r_{2} b_{11} b_{32}-r_{3} b_{11} b_{22}}{b_{11} b_{22} b_{33}} .
$$

Proof. From the results in [16] and if Assumption 3.5 is satisfied, we know

$$
\lim _{t \rightarrow \infty} \frac{\log \Phi_{1}(t)}{t}=0, \quad \lim _{t \rightarrow \infty} \frac{1}{t} \int_{0}^{t} \Phi_{1}(s) d s=\frac{a_{1}-\frac{\sigma_{1}^{2}}{2}}{b_{11}}=\frac{r_{1}}{b_{11}}=M_{1}, \quad \text { a.s., }
$$

besides, according to Itô's formula, the second population of system (2.2) is changed into

$$
d \log \Phi_{2}(t)=\left(-r_{2}+b_{21} \Phi_{1}(t-\tau)-b_{22} \Phi_{2}(t)\right) d t-\sigma_{2} d B_{2}(t) .
$$

It then follows

$$
\log \Phi_{2}(t)=\log \Phi_{2}(0)-r_{2} t+b_{21} \int_{0}^{t} \Phi_{1}(s-\tau) d s-b_{22} \int_{0}^{t} \Phi_{2}(s) d s-\sigma_{2} B_{2}(t) .
$$


Notice that

$$
\int_{0}^{t} \Phi_{1}(s-\tau) d s=\int_{-\tau}^{t-\tau} \Phi_{1}(s) d s=\int_{-\tau}^{0} \xi_{1}(s) d s+\int_{0}^{t} \Phi_{1}(s) d s-\int_{t-\tau}^{t} \Phi_{1}(s) d s
$$

and from the second equation of (3.1), we get $\lim _{t \rightarrow \infty} \frac{1}{t} \int_{t-\tau}^{t} \Phi_{1}(s) d s=0$, dividing the equation (3.3) both sides by $t$, and taking $t \rightarrow \infty$, yields

$$
\lim _{\mathrm{t} \rightarrow \infty} \frac{1}{\mathrm{t}} \int_{0}^{\mathrm{t}} \Phi_{1}(s-\tau) \mathrm{d} s=\lim _{\mathrm{t} \rightarrow \infty} \frac{1}{\mathrm{t}} \int_{0}^{\mathrm{t}} \Phi_{1}(\mathrm{~s}) \mathrm{d} s=\mathrm{M}_{1}
$$

so

$$
\lim _{t \rightarrow 0} \frac{\log \Phi_{2}(0)-r_{2} t+b_{21} \int_{0}^{t} \Phi_{1}(s-\tau) d s-\sigma_{2} B_{2}(t)}{t}=-r_{2}+b_{21} \frac{r_{1}}{b_{11}} .
$$

With Lemma 3.4 and Assumption 3.5 we could get

$$
\lim _{t \rightarrow \infty} \frac{1}{t} \int_{0}^{t} \Phi_{2}(s) d s=\frac{-r_{2}+b_{21} \frac{r_{1}}{b_{11}}}{b_{22}}=\frac{r_{1} b_{21}-r_{2} b_{11}}{b_{11} b_{22}}=M_{2}>0 .
$$

Let (3.2) divide $t$, and $t \rightarrow \infty$, together with (3.1) and (3.3), consequently

$$
\lim _{t \rightarrow \infty} \frac{\log \Phi_{2}(t)}{t}=0 .
$$

Similarly, according to Ito's formula, the third population of system (2.2) is changed into

$$
d \log \Phi_{3}(t)=\left(-r_{3}+b_{32} \Phi_{2}(t-\tau)-b_{33} \Phi_{3}(t)\right) d t-\sigma_{3} d B_{3}(t),
$$

it then follows

$$
\log \Phi_{3}(t)=\log \Phi_{3}(0)-r_{3} t+b_{32} \int_{0}^{t} \Phi_{2}(s-\tau) d s-b_{33} \int_{0}^{t} \Phi_{3}(s) d s-\sigma_{3} B_{3}(t)
$$

and

$$
\lim _{t \rightarrow \infty} \frac{1}{t} \int_{0}^{t} \Phi_{3}(s) d s=\frac{-r_{3}+b_{32} \frac{r_{1} b_{21}-r_{2} b_{11}}{b_{11} b_{22}}}{b_{33}}=M_{3}>0, \quad \lim _{t \rightarrow \infty} \frac{\log \Phi_{3}(t)}{t}=0 .
$$

From this, together with Theorem 2.2 and Lemma 3.6, the following result is obviously true.

Theorem 3.7. If Assumption 3.5 is satisfied, then the solution $x(t, \xi)$ of system (1.3) has the following property:

$$
\limsup _{t \rightarrow \infty} \frac{\log x_{i}(t)}{t} \leqslant 0, \quad i=1,2,3 .
$$

By above all, we could get the following result.

Theorem 3.8. If Assumption 3.5 is satisfied, then the solution $x(t, \xi)$ of system (1.3) has the following property

$$
\liminf _{t \rightarrow \infty} \frac{1}{t} \int_{0}^{t} x_{3}(s) d s \geqslant \tilde{x}_{3}^{*} \text {, a.s., }
$$

where $\tilde{x}^{*}=\left(\tilde{x}_{1}^{*}, \tilde{x}_{2}^{*}, \tilde{x}_{3}^{*}\right)$ is the only nonnegative solution of the following equation,

$$
\left\{\begin{array}{l}
r_{1}-b_{11} x_{1}-b_{12} x_{2}=0 \\
-r_{2}+b_{21} x_{1}-b_{22} x_{2}-b_{23} x_{3}=0 \\
-r_{3}+b_{32} x_{2}-b_{33} x_{3}=0
\end{array}\right.
$$


Proof. From system (1.3),

$$
\begin{aligned}
\frac{\log x_{1}(t)-\log x_{1}(0)}{t}= & r_{1}-b_{11} \frac{1}{t} \int_{0}^{t} x_{1}(s) d s-b_{12} \frac{1}{t} \int_{0}^{t} x_{2}(s-\tau) d s+\frac{\sigma_{1} B_{1}(t)}{t} \\
= & r_{1}-b_{11} \frac{1}{t} \int_{0}^{t} x_{1}(s) d s-b_{12} \frac{1}{t}\left(\int_{-\tau}^{0} \xi_{2}(s) d s-\int_{t-\tau}^{t} x_{2}(s) d s\right) \\
& -b_{12} \frac{1}{t} \int_{0}^{t} x_{2}(s) d s+\frac{\sigma_{1} B_{1}(t)}{t}
\end{aligned}
$$

similarly,

$$
\begin{aligned}
\frac{\log x_{2}(t)-\log x_{2}(0)}{t}= & -r_{2}+b_{21} \frac{1}{t} \int_{0}^{t} x_{1}(s) d s+b_{21}\left(\frac{1}{t} \int_{-\tau}^{0} \xi_{1}(s) d s-\frac{1}{t} \int_{t-\tau}^{t} x_{1}(s) d s\right)-b_{22} \frac{1}{t} \int_{0}^{t} x_{2}(s) d s \\
& -b_{23} \frac{1}{t} \int_{0}^{t} x_{3}(s) d s-b_{23}\left(\frac{1}{t} \int_{-\tau}^{0} \xi_{3}(s) d s-\frac{1}{t} \int_{t-\tau}^{t} x_{3}(s) d s\right)-\frac{\sigma_{2} B_{2}(t)}{t}
\end{aligned}
$$

and

$$
\begin{aligned}
\frac{\log x_{3}(t)-\log x_{3}(0)}{t}= & -r_{3}+b_{32} \frac{1}{t} \int_{0}^{t} x_{2}(s) d s+b_{32}\left(\frac{1}{t} \int_{-\tau}^{0} \xi_{2}(s) d s-\frac{1}{t} \int_{t-\tau}^{t} x_{2}(s) d s\right) \\
& -b_{33} \frac{1}{t} \int_{0}^{t} x_{3}(s) d s-\frac{\sigma_{3} B_{3}(t)}{t} .
\end{aligned}
$$

Hence

$$
\begin{aligned}
& \frac{c_{1}\left(\log x_{1}(t)-\log x_{1}(0)\right)+c_{2}\left(\log x_{2}(t)-\log x_{2}(0)\right)+c_{3}\left(\log x_{3}(t)-\log x_{3}(0)\right)}{t} \\
& =\left(r_{1} c_{1}-r_{2} c_{2}-r_{3} c_{3}\right)+\left(-b_{11} c_{1}+b_{21} c_{2}\right) \frac{1}{t} \int_{0}^{t} x_{1}(s) d s \\
& \quad+\left(-b_{12} c_{1}-b_{22} c_{2}+b_{32} c_{3}\right) \frac{1}{t} \int_{0}^{t} x_{2}(s) d s-\left(b_{23} c_{2}+b_{33} c_{3}\right) \frac{1}{t} \int_{0}^{t} x_{3}(s) d s \\
& \quad-c_{1} b_{12} \frac{1}{t}\left(\int_{-\tau}^{0} \xi_{2}(s) d s-\int_{t-\tau}^{t} x_{2}(s) d s\right)+c_{2} b_{21}\left(\frac{1}{t} \int_{-\tau}^{0} \xi_{1}(s) d s-\frac{1}{t} \int_{t-\tau}^{t} x_{1}(s) d s\right) \\
& \quad-c_{2} b_{23}\left(\frac{1}{t} \int_{-\tau}^{0} \xi_{3}(s) d s-\frac{1}{t} \int_{t-\tau}^{t} x_{3}(s) d s\right)+c_{3} b_{32}\left(\frac{1}{t} \int_{-\tau}^{0} \xi_{2}(s) d s-\frac{1}{t} \int_{t-\tau}^{t} x_{2}(s) d s\right) \\
& \quad+\frac{c_{1} \sigma_{1} B_{1}(t)}{t}-\frac{c_{2} \sigma_{2} B_{2}(t)}{t}-\frac{c_{3} \sigma_{3} B_{3}(t)}{t} .
\end{aligned}
$$

From Theorem 2.2, we get

$$
x_{i}(t) \leqslant \Phi_{i}(t), \quad(i=1,2,3),
$$

then

$$
\lim _{t \rightarrow \infty} \frac{1}{t} \int_{t-\tau}^{t} x_{i}(s) d s=0 .
$$

Let $c_{1}=b_{21}, c_{2}=b_{11}, c_{3}=\frac{b_{11} b_{22}+b_{12} b_{21}}{b_{32}}$, together with Assumption 3.5, we know

$$
r_{1} c_{1}-r_{2} c_{2}-r_{3} c_{3}>0
$$


According to Theorem 3.7 and equation (3.5), together with $\lim _{t \rightarrow \infty} \frac{B_{i}(t)}{t}=0,(i=1,2,3)$, we could get

$$
\begin{gathered}
\limsup _{t \rightarrow \infty} \frac{c_{1}\left(\log x_{1}(t)-\log x_{1}(0)\right)+c_{2}\left(\log x_{2}(t)-\log x_{2}(0)\right)+c_{3}\left(\log x_{3}(t)-\log x_{3}(0)\right)}{t} \\
=\left(r_{1} c_{1}-r_{2} c_{2}-r_{3} c_{3}\right)-\left(c_{2} b_{23}+c_{3} b_{33}\right) \liminf _{t \rightarrow \infty} \frac{1}{t} \int_{0}^{t} x_{3}(s) d s \leqslant 0 .
\end{gathered}
$$

Such that,

$$
\liminf _{t \rightarrow \infty} \frac{1}{t} \int_{0}^{t} x_{3}(s) d s \geqslant \frac{r_{1} c_{1}-r_{2} c_{2}-r_{3} c_{3}}{c_{2} b_{23}+c_{3} b_{33}}=\tilde{x}_{3}^{*}
$$

where $\tilde{x}^{*}=\left(\tilde{x}_{1}^{*}, \tilde{x}_{2}^{*}, \tilde{x}_{3}^{*}\right)$ is the only nonnegative solution of the following equation when Assumption 3.5 is satisfied,

$$
\left\{\begin{array}{l}
r_{1}-b_{11} x_{1}-b_{12} x_{2}=0 \\
-r_{2}+b_{21} x_{1}-b_{22} x_{2}-b_{23} x_{3}=0 \\
-r_{3}+b_{32} x_{2}-b_{33} x_{3}=0
\end{array}\right.
$$

\section{Non-persistence}

In the previous section, we showed the solution $x(t, \xi)$ of system (1.3) is stable in time average, and in this section, we discuss the dynamics of system (1.3) when the white noise is getting larger. We show the situation when the population of system (1.3) will be non-persistent of the white noise is large, which does not happen in the deterministic system in three cases.

Definition 4.1. System (1.3) is said to be non-persistent, if there are positive constants $c_{i},(i=1,2,3)$ such that

$$
\lim _{t \rightarrow \infty} \prod_{i=1}^{3} x_{i}^{c_{i}}(t)=0, \text { a.s.. }
$$

Now we present conditions for all species or some species of (1.3) to be extinct. Consider the following cases.

Case (i): $r_{1}<0$.

According to Itô's formula, the first population of system (2.3) is changed into

$$
d \log \Phi_{1}(t) \leqslant\left(r_{1}-b_{11} \Phi_{1}(t)\right) d t-\sigma_{1} d B_{1}(t) .
$$

If $r_{1}<0$, we could get

$$
\limsup _{t \rightarrow \infty} \frac{\log \Phi_{1}(t)}{t}=r_{1}<0 \text { a.s., }
$$

from the stochastic comparison theorem, we have

$$
\limsup _{t \rightarrow \infty} \frac{\log x_{1}(t)}{t}<0 \text { a.s., }
$$

hence

$$
\lim _{t \rightarrow \infty} x_{1}(t)=0 \text {, a.s.. }
$$


From the second population of system (2.3) and equation (3.3), we have

$$
\frac{\log \Phi_{2}(\mathrm{t})-\log \Phi_{2}(0)}{\mathrm{t}} \leqslant-\mathrm{r}_{2}+\mathrm{b}_{21} \frac{1}{\mathrm{t}} \int_{0}^{\mathrm{t}} \Phi_{1}(s-\tau) \mathrm{d} s-\frac{\sigma_{2} \mathrm{~dB}_{2}(\mathrm{t})}{\mathrm{t}} \text { a.s. },
$$

hence

$$
\begin{aligned}
\limsup _{t \rightarrow \infty} \frac{\log \Phi_{2}(t)}{t} & \leqslant-r_{2}+b_{21} \limsup _{t \rightarrow \infty} \frac{1}{t} \int_{0}^{t} \Phi_{1}(s-\tau) d s \\
& =-r_{2}+b_{21} \limsup _{t \rightarrow \infty} \frac{1}{t} \int_{0}^{t} \Phi_{1}(s) d s=-r_{2} \leqslant 0 \text {, a.s.. }
\end{aligned}
$$

Similarly,

$$
\limsup _{t \rightarrow \infty} \frac{\log \Phi_{3}(t)}{t}=-r_{3} \leqslant 0, \text { a.s., }
$$

and

$$
\lim _{t \rightarrow \infty} x_{i}(t)=0 \text {, a.s. } i=2,3 \text {. }
$$

Case (ii): $r_{1}>0, r_{1}-\frac{b_{11}}{b_{21}} r_{2}<0$.

It is clear that from the equations (4.1) and (3.1), we get

$$
\limsup _{t \rightarrow \infty} \frac{\log \Phi_{2}(t)}{t} \leqslant-r_{2}+b_{21} \frac{r_{1}}{b_{11}}<0, \text { a.s.. }
$$

Similarly

$$
\limsup _{t \rightarrow \infty} \frac{\log \Phi_{3}(t)}{t} \leqslant-r_{3}+b_{32} \limsup _{t \rightarrow \infty} \frac{1}{t} \int_{0}^{t} \Phi_{2}(s) d s=-r_{3}<0 \text {, a.s., }
$$

thus,

$$
\lim _{t \rightarrow \infty} x_{i}(t)=0 \text {, a.s., } i=2,3 \text {. }
$$

By above all, and from the conclusion in [14], we could easily know that the distribution of $x_{1}(t)$ converges weekly to the probability measure with density

$$
f^{*}(\zeta)=C_{0} \zeta^{2 r_{1} / \sigma_{1}^{2}-1} e^{-2 b_{11} \zeta / \sigma_{1}^{2}},
$$

where $C_{0}=\left(2 b_{11} / \sigma_{1}^{2}\right)^{2 r_{1} / \sigma_{1}^{2}} / \Gamma\left(2 r_{1} / \sigma_{1}^{2}\right)$, and

$$
\lim _{t \rightarrow \infty} \frac{1}{t} \int_{0}^{t} x_{1}(s) d s=\frac{r_{1}}{b_{11}}, \text { a.s.. }
$$

Case (iii): $r_{1}-\frac{b_{11}}{b_{21}} r_{2}-\frac{b_{11} b_{22}+b_{12} b_{21}}{b_{21} b_{32}} r_{3}<0$. we get

It is clear that from (3.4), letting $c_{1}=b_{21}, c_{2}=b_{11}, c_{3}=\frac{b_{11} b_{22}+b_{12} b_{21}}{b_{32}}$, with $\lim _{t \rightarrow \infty} \frac{B_{i}(t)}{t}=0, i=1,2,3$,

$$
\begin{aligned}
& \limsup _{t \rightarrow \infty} \frac{c_{1}\left(\log x_{1}(t)-\log x_{1}(0)\right)+c_{2}\left(\log x_{2}(t)-\log x_{2}(0)\right)+c_{3}\left(\log x_{3}(t)-\log x_{3}(0)\right)}{t} \\
& \quad=\left(r_{1} c_{1}-r_{2} c_{2}-r_{3} c_{3}\right)-\left(c_{2} b_{23}+c_{3} b_{33}\right) \liminf _{t \rightarrow \infty} \frac{1}{t} \int_{0}^{t} x_{3}(s) d s \\
& \quad \leqslant r_{1} c_{1}-r_{2} c_{2}-r_{3} c_{3}
\end{aligned}
$$


moreover,

$$
\limsup _{t \rightarrow \infty} \frac{\log x_{1}^{c_{1}}(t) x_{2}^{c_{2}}(t) x_{3}^{c_{3}}(t)}{t} \leqslant r_{1} c_{1}-r_{2} c_{2}-r_{3} c_{3}<0
$$

then

$$
\lim _{t \rightarrow \infty} x_{1}^{c_{1}}(t) x_{2}^{c_{2}}(t) x_{3}^{c_{3}}(t)=0, \text { a.s.. }
$$

Therefore, by the above arguments, we get the following conclusion.

Theorem 4.2. Let $x(t, \xi)$ be the solution of system (1.3), the following conclusions are founded.

(1) If $r_{1}<0$, then

$$
\lim _{t \rightarrow \infty} x_{i}(t)=0, \text { a.s., } i=1,2,3 \text {. }
$$

(2) If $r_{1}>0, r_{1}-\frac{b_{11}}{b_{21}} r_{2}<0$, then

$$
\lim _{t \rightarrow \infty} x_{i}(t)=0, \text { a.s., } i=2,3,
$$

and the distribution of $x_{1}(t)$ converges weekly to the probability measure with density

$$
f^{*}(\zeta)=C_{0} \zeta^{2 r_{1} / \sigma_{1}^{2}-1} e^{-2 b_{11} \zeta / \sigma_{1}^{2}}
$$

where $\mathrm{C}_{0}=\left(2 \mathrm{~b}_{11} / \sigma_{1}^{2}\right)^{2 \mathrm{r}_{1} / \sigma_{1}^{2}} / \Gamma\left(2 \mathrm{r}_{1} / \sigma_{1}^{2}\right)$, and

$$
\lim _{t \rightarrow \infty} \frac{1}{t} \int_{0}^{t} x_{1}(s) d s=\frac{r_{1}}{b_{11}}, \text { a.s.. }
$$

(3) If $r_{1}-\frac{b_{11}}{b_{21}} r_{2}-\frac{b_{11} b_{22}+b_{12} b_{21}}{b_{21} b_{32}} r_{3}<0$, then

$$
\lim _{t \rightarrow \infty} x_{1}^{c_{1}}(t) x_{2}^{c_{2}}(t) x_{3}^{c_{3}}(t)=0, \text { a.s., }
$$

where $c_{1}=b_{21}, c_{2}=b_{11}, c_{3}=\frac{b_{11} b_{22}+b_{12} b_{21}}{b_{32}}$.

That is to say, the large white noise will lead to the population system non-persistent.

\section{Numerical simulation}

In this section, we give out the numerical experiment to support our results. Consider the equation

$$
\left\{\begin{array}{l}
d x_{1}(t)=x_{1}(t)\left(a_{1}-b_{11} x_{1}(t)-b_{12} x_{2}(t-\tau)\right) d t+\sigma_{1} x_{1}(t) d B_{1}(t), \\
d x_{2}(t)=x_{2}(t)\left(-a_{2}+b_{21} x_{1}(t-\tau)-b_{22} x_{2}(t)-b_{23} x_{3}(t-\tau)\right) d t-\sigma_{2} x_{2}(t) d B_{2}(t), \\
d x_{3}(t)=x_{3}(t)\left(-a_{3}+b_{32} x_{2}(t-\tau)-b_{33} x_{3}(t)\right) d t-\sigma_{3} x_{3}(t) d B_{3}(t) .
\end{array}\right.
$$

By the method in [11], we have the difference equation

$$
\left\{\begin{array}{l}
x_{1, k+1}=x_{1, k}+x_{1, k}\left[\left(a_{1}-b_{11} x_{1, k}-b_{12} x_{2, k-m}\right) \Delta t+\sigma_{1} \epsilon_{1, k} \sqrt{\Delta} t+\frac{\sigma_{1}^{2}}{2}\left(\epsilon_{1, k}^{2} \Delta t-\Delta t\right)\right], \\
x_{2, k+1}=x_{2, k}+x_{2, k}\left[\left(-a_{2}+b_{21} x_{1, k-m}-b_{22} x_{2, k}-b_{23} x_{3, k-m}\right) \Delta t-\sigma_{2} \epsilon_{2, k} \sqrt{\Delta} t+\frac{\sigma_{2}^{2}}{2}\left(\epsilon_{2, k}^{2} \Delta t-\Delta t\right)\right], \\
x_{3, k+1}=x_{3, k}+x_{3, k}\left[\left(-a_{3}+b_{32} x_{2, k-m}-b_{33} x_{3, k}\right) \Delta t-\sigma_{3} \epsilon_{3, k} \sqrt{\Delta} t+\frac{\sigma_{3}^{2}}{2}\left(\epsilon_{3, k}^{2} \Delta t-\Delta t\right)\right],
\end{array}\right.
$$


where $\epsilon_{1, k}, \epsilon_{2, k}$ and $\epsilon_{3, k}, i=1,2,3$ are the Gaussian random variables $N(0,1), r_{1}=a_{1}-\frac{\sigma_{1}^{2}}{2}>0, r_{i}=a_{i}+$ $\frac{\sigma_{i}^{2}}{2},(i=2,3)$, and $m$ represents the integer part $\tau / \triangle t-1$. Choose $\left(x_{1}(0), x_{2}(0), x_{3}(0)\right) \in R_{+}^{3}, t \in[-\tau, 0]$, and suitable parameters, by Matlab, we get Figs. 1, 2, and 3.
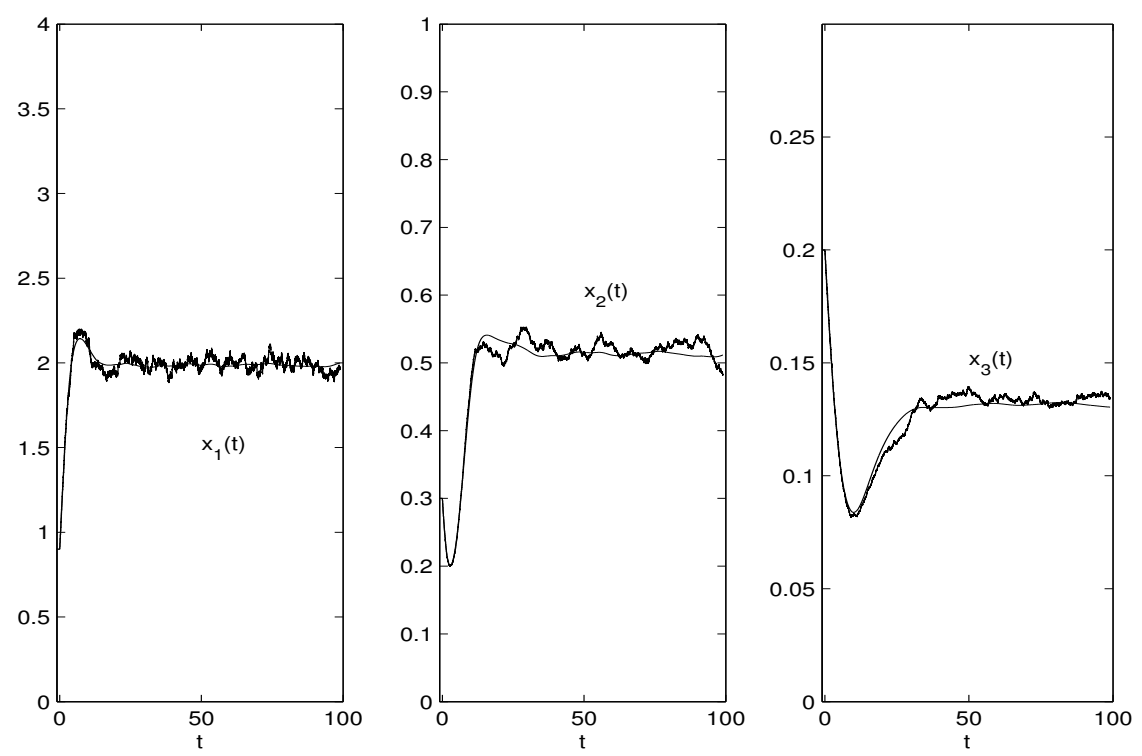

Figure 1: The solutions of system (1.2) and system (1.3) with $\left(x_{1}(0), x_{2}(0), x_{3}(0)\right)=(0.9,0.3,0.2), t \in[-\tau, 0], a_{1}=0.7, a_{2}=$ $0.3, a_{3}=0.1, b_{11}=0.3, b_{12}=0.2, b_{21}=0.3, b_{22}=0.5, b_{23}=0.3, b_{32}=0.4, b_{33}=0.8$. The light lines represent the solution of system (1.2), while the dark lines represent the solution of system (1.3) with $\sigma_{1}=0.02, \sigma_{2}=0.01, \sigma_{3}=0.01$.
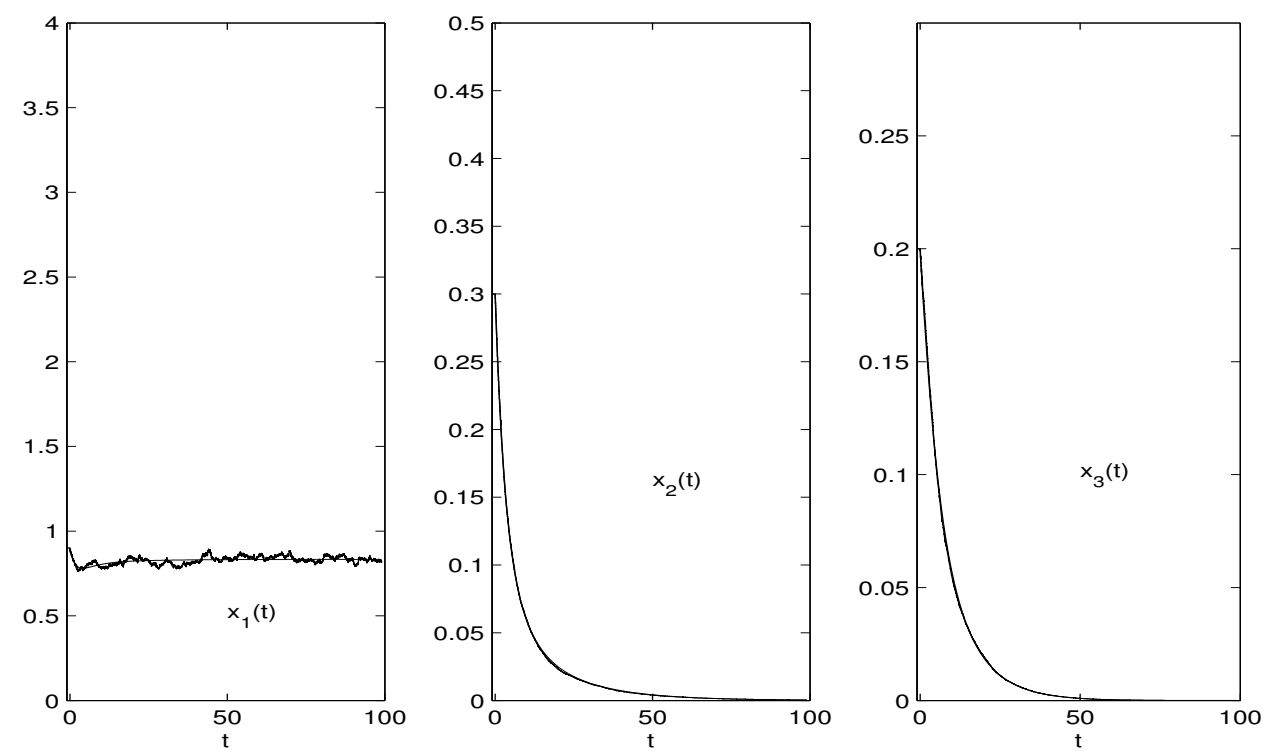

Figure 2: Two of the species will die out in probability. The solutions of system (1.2) and system $(1.3)$ with $\left(x_{1}(0), x_{2}(0), x_{3}(0)\right)=$ $(0.9,0.3,0.2), t \in[-\tau, 0], a_{1}=0.5, a_{2}=0.3, a_{3}=0.1, b_{11}=0.6, b_{12}=0.2, b_{21}=0.3, b_{22}=0.5, b_{23}=0.3, b_{32}=0.4, b_{33}=0.8$. The light lines represent the solution of system (1.2), while the dark lines represent the solution of system (1.3) with $\sigma_{1}=$ $0.02, \sigma_{2}=0.01, \sigma_{3}=0.01$. 

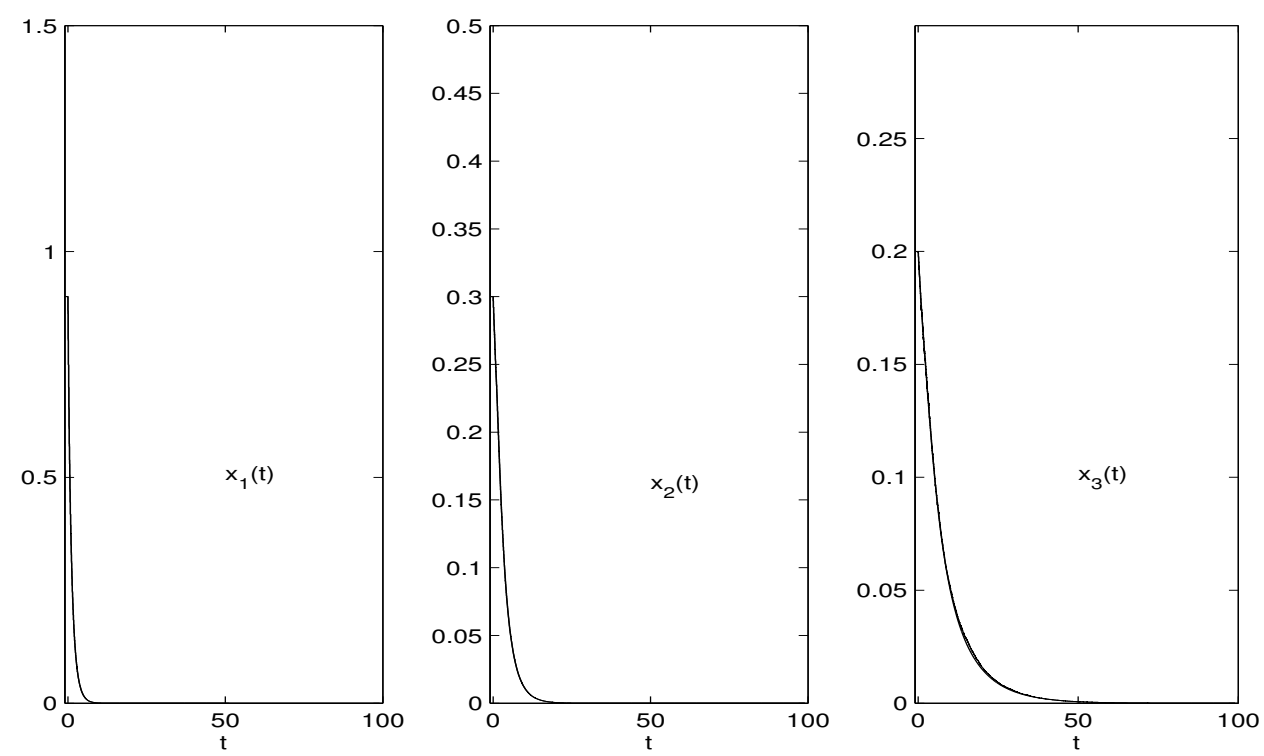

Figure 3: One of the species or both species will die out in probability. The solutions of system (1.2) and system (1.3) with $\left(x_{1}(0), x_{2}(0), x_{3}(0)\right)=(0.9,0.3,0.2), t \in[-\tau, 0], a_{1}=-0.7 a_{2}=0.3, a_{3}=0.1, b_{11}=0.3, b_{12}=0.2, b_{21}=0.3, b_{22}=0.5, b_{23}=$ $0.3, b_{32}=0.4, b_{33}=0.8$. The light lines represent the solution of system (1.2), while the dark lines represent the solution of system (1.3) with $\sigma_{1}=0.02, \sigma_{2}=0.01, \sigma_{3}=0.01$.

In Fig. 1, when the noise is small, choose parameters satisfying the condition of Theorem 3.7, then the solution of system (1.2) will persist in time average.

In Fig. 2, we observe Case (iii) in Theorem 4.2 and choose parameters $r_{1}>0, r_{1}-\frac{b_{11}}{b_{21}} r_{2}<0$. As Theorem 4.2 indicated that two predators will die out in probability, then the prey solution of system (1.2) will persist in time average.

In Fig. 3, we observe Case (i) in Theorem 4.2 and choose parameters $r_{1}<0$. As Theorem 4.2 indicated that not only predators but also prey will die out in probability when the noise of the prey is large, and it does not happen in the deterministic system, these simulated results are consistent with our theorems.

\section{Acknowledgment}

The work was supported by Natural Science Foundation of Jilin Province of China (No. 201115133).

\section{References}

[1] M. Barra, G. Del Grosso, A. Gerardi, G. Koch, F. Marchetti, Some basic properties of stochastic population models, Systems theory in immunology, Proc. Working Conf., Rome, (1978), Lecture Notes in Biomath., Springer, BerlinNew York, 32 (1979), 155-164. 1

[2] L.-S. Chen, J. Chen, Nonlinear biological dynamical system, Science Press, Beijing, (1993). 3

[3] H. I. Freedman, Deterministic mathematical models in population ecology, Monographs and Textbooks in Pure and Applied Mathematics, Marcel Dekker, Inc., New York, (1980). 1

[4] H. I. Freedman, K. Gopalsamy, Global stability in time-delayed single-species dynamics, Bull. Math. Biol., 48 (1986), 485-492. 1

[5] H. I. Freedman, J. W.-H. So, Global stability and persistence of simple food chains, Math. Biosci., 76 (1985), 69-86. 1

[6] H. I. Freedman, P. Waltman, Mathematical analysis of some three-species food-chain models, Math. Biosci., 33 (1977), 257-276. 1

[7] T. C. Gard, Persistence in stochastic food web models, Bull. Math. Biol., 46 (1984), 357-370. 1

[8] T. C. Gard, Stability for multispecies population models in random environments, Nolinear Anal., 10 (1986), 1411-1419. 1,1

[9] T. C. Gard, Introduction to stochastic differential equations, Monographs and Textbooks in Pure and Applied Mathematics, Marcel Dekker, Inc., New York, (1988). 1, 1 
[10] K. Gopalsamy, Global asymptotic stability in a periodic Lotka-Volterra system, J. Austral. Math. Soc. Ser. B, 24 (1982), 66-72. 1

[11] D. J. Higham, An algorithmic introduction to numerical simulation of stochastic differential equations, SIAM Rev., 43 (2001), 525-546. 5

[12] Y.-Z. Hu, F.-K. Wu, C.-M. Huang, Stochastic Lotka-Volterra models with multiple delays, J. Math. Anal. Appl., 375 (2011), 42-57. 1

[13] N. Ikeda, S. Watanabe, Stochastic differential equations and diffusion processes, North-Holland Mathematical Library, North-Holland Publishing Co., Amsterdam-New York; Kodansha, Ltd., Tokyo, (1981). 1

[14] C.-Y. Ji, D.-Q. Jiang, Dynamics of a stochastic density dependent predator-prey system with Beddington-DeAngelis functional response, J. Math. Anal. Appl., 381 (2011), 441-453. 1, 4

[15] C.-Y. Ji, D.-Q. Jiang, X.-Y. Li, Qualitative analysis of a stochastic ratio-dependent predator-prey system, J. Comput. Appl. Math., 235 (2011), 1326-1341. 1

[16] C.-Y. Ji, D.-Q. Jiang, N.-Z. Shi, Analysis of a predator-prey model with modified Leslie-Gower and Holling-type II schemes with stochastic perturbation, J. Math. Anal. Appl., 359 (2009), 482-498. 1, 3

[17] C.-Y. Ji, D.-Q. Jiang, N.-Z. Shi, D. O’Regan, Existence, uniqueness, stochastic persistence and global stability of positive solutions of the logistic equation with random perturbation, Math. Methods Appl. Sci., 30 (2007), 77-89. 1

[18] D.-Q. Jiang, N.-Z. Shi, A note on nonautonomous logistic equation with random perturbation, J. Math. Anal. Appl., 303 (2005), 164-172. 2.1

[19] Y. Kuang, Delay differential equations with applications in population dynamics, Mathematics in Science and Engineering, Academic Press, Inc., Boston, MA, (1993). 1

[20] Y. Kuang, H. L. Smith, Global stability for infinite delay Lotka-Volterra type systems, J. Differential Equations, 103 (1993), 221-246. 1

[21] H.-H. Li, F.-Z. Cong, D.-Q. Jiang, H.-T. Hua, Persistence and nonpersistence of a food chain model with stochastic perturbation, Abstr. Appl. Anal., 2013 (2013), 9 pages. 1

[22] X.-R. Mao, Stochastic differential equations and their applications, Horwood Publishing Series in Mathematics \& Applications, Horwood Publishing Limited, Chichester, (1997). 1, 2

[23] X.-R. Mao, Delay population dynamics and environmental noise, Stoch. Dyn., 5 (2005), 149-162. 1

[24] X.-R. Mao, C.-G. Yuan, J.-Z. Zou, Stochastic differential delay equations of population dynamics, J. Math. Anal. Appl., 304 (2005), 296-320. 1, 1

[25] R. M. May, Stability and complexity in model ecosystems, Princeton university press, New Jersey, (2001). 1

[26] P. Polansky, Invariant distributions for multipopulation models in random environments, Theoret. Population Biol., 16 (1979), 25-34. 1

[27] P.-Y. Xia, X.-K. Zheng, D.-Q. Jiang, Persistence and nonpersistence of a nonautonomous stochastic mutualism system, Abstr. Appl. Anal., 2013 (2013), 13 pages. 3.2

[28] W. Xiaoqing, M. Zhien, H. I. Freedman, Global stability of Volterra models with time delay, J. Math. Anal. Appl., 160 (1991), 51-59. 1

[29] C. Zhu, G. Yin, On competitive Lotka-Volterra model in random environments, J. Math. Anal. Appl., 357 (2009), 154-170. 1 